Supporting Information

\title{
Hierarchical Porous Carbon Nanofibers with Tunable Geometries and Porous Structures Fabricated by a Scalable Electrospinning Technique
}

Shuhui Xia", Yuanyuan Zhang", Yun Zhao, Xiao Wang and Jianhua Yan*

S. Xia, Y. Zhang, Y. Zhao, and Prof. J. Yan

Key Laboratory of Textile Science \& Technology, College of Textile, Donghua

University, Shanghai 201620, China

Corresponding authors: Prof. Jianhua Yan

E-mail: yanjianhua@dhu.edu.cn

Prof. J. Yan

School of Textile Materials and Engineering, Wuyi University, Guangdong 529020,

China

X. Wang,

College of Materials Science and Engineering, Donghua University, Shanghai

201620, China 


\section{Supplementary Tables}

Supplementary Table 1. Elemental analysis of PCNFs@S composites by inductively coupled plasma atomic emission analyzer.

\begin{tabular}{cccccc}
\hline & C & H & N & B & S \\
Sample & $(w t \%)$ & $(w t \%)$ & $(w t \%)$ & $(w t \%)$ & $(w t \%)$ \\
\hline PCNF@S & 10.86 & 0.31 & $\leq 0.05$ & 0.21 & 85.35 \\
\hline
\end{tabular}




\section{Supplementary Figures}

(a)

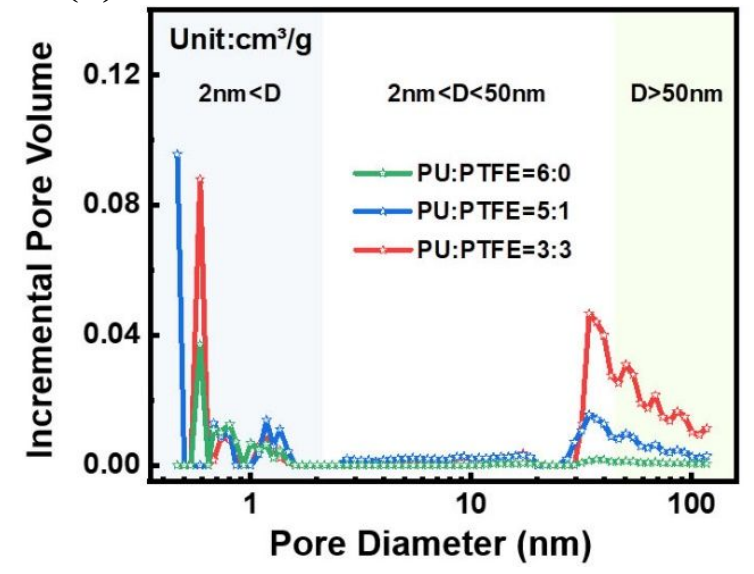

(b)

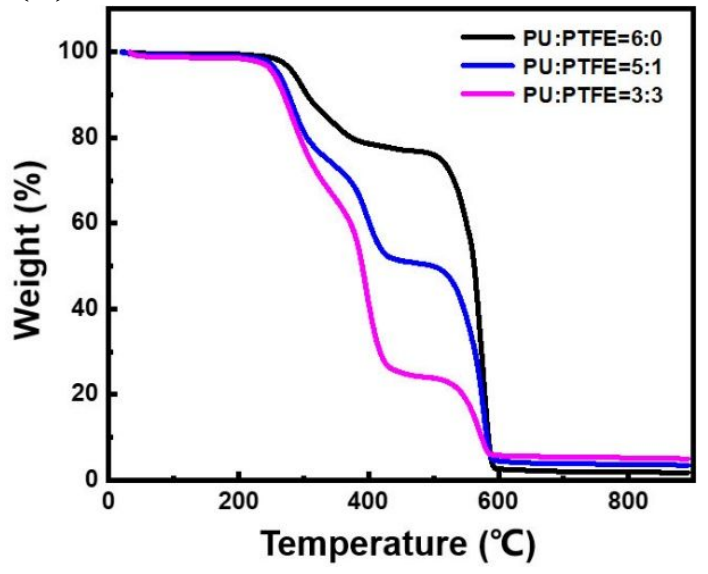

Figure S1. (a) The incremental pore volume and (b) TG analysis of different samples.

(a) Here, the incremental pore volume of different samples was calculated by $\mathrm{N}_{2}$-DFT model. By accumulating the pore volume under different pore sizes, we learned the influence of the mass ratio of PU and PTFE on the pore volume. When the ratios of PU/PTFE were 6:0, 5:1, and 3:3, the volume fraction of micropores gradually decreased from $91.0 \%$ to $23.0 \%$, while the mesopores gradually increased from $5.6 \%$ to $46.6 \%$ and the macropores also increased from $3.4 \%$ to $30.4 \%$. (b) The TG tests were conducted in $\mathrm{N}_{2}$-atmosphere at a heating rate of $5^{\circ} \mathrm{C} \mathrm{min}^{-1}$. When the ratios of PU/PTFE were $6: 0,5: 1$, and 3:3, the total carbon yield increased continuously. However, the three samples have very low yields after carbonization, and the maximum value is only $5.2 \%$ according to the TG analysis. 

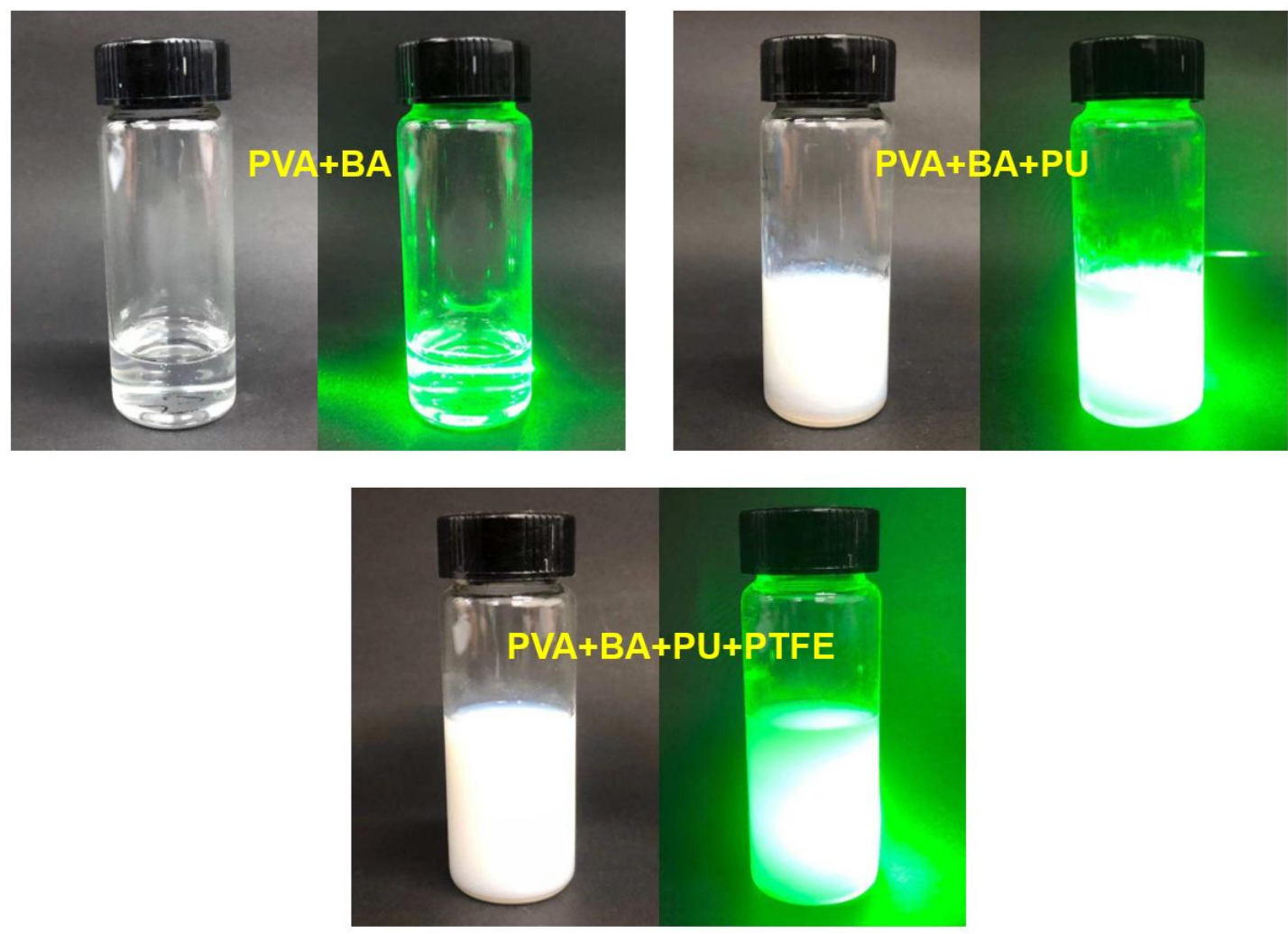

Figure S2. Optical images and Tyndall effect of the precursor containing various materials.

If the solution system only contained PVA and BA, BA chemically reacted with PVA to form borate diol bonds. At this time, a bright path in the sol could be observed under a beam of light, which proved that the precursor solution was a sol system. However, when introducing PU (Polyurethane emulsion) and PTFE NPs, the transparent sol gradually turned into an opaque emulsion with a certain viscosity, which indicated that PU and PFTE existed in the precursor in their initial form, respectively. 


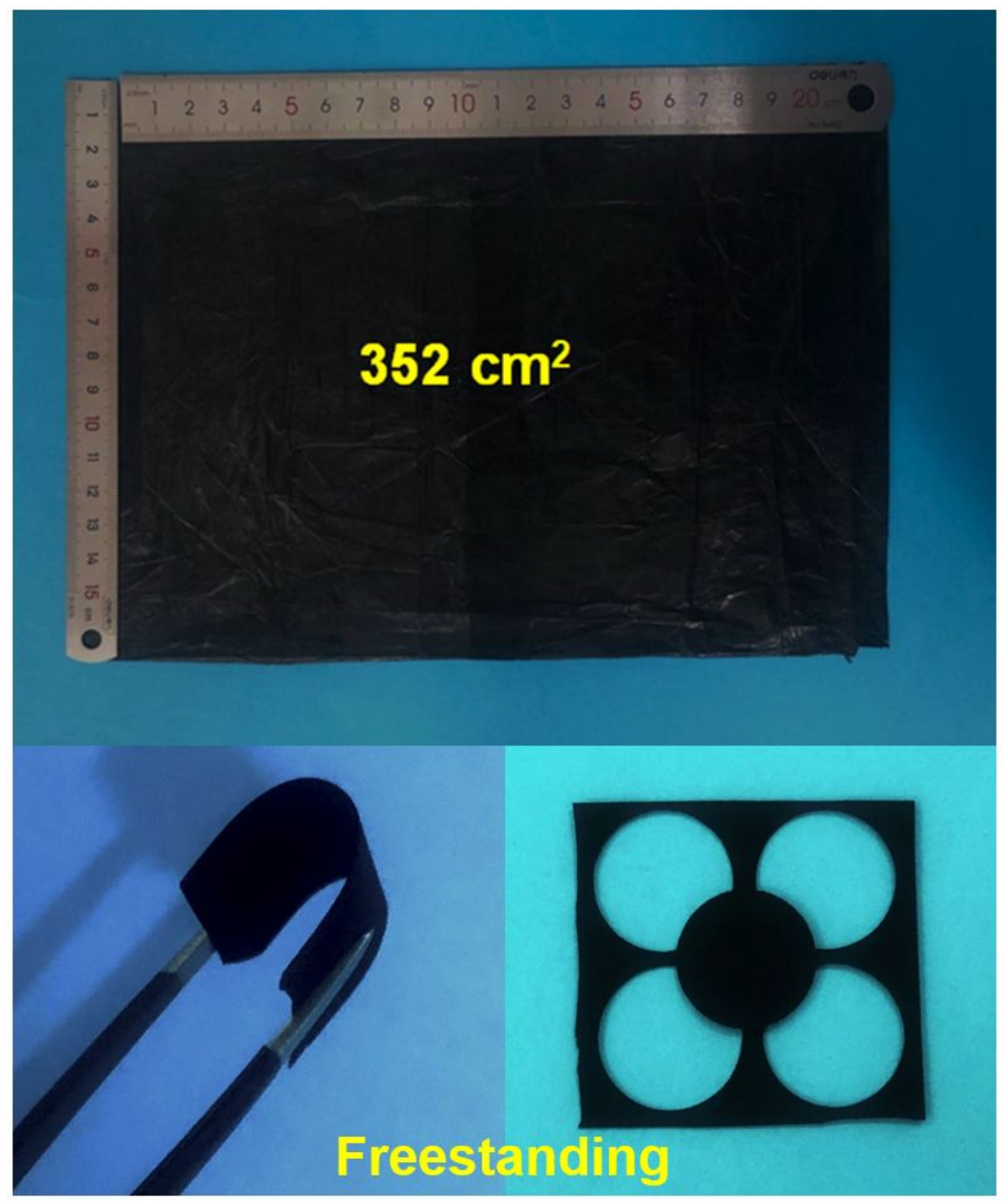

Figure S3. A large scale PCNF film and the demonstration of its mechanical

\section{flexibility.}

As we know, the area of PCNF films could be well controlled by simply adjusting the collector areas of electrospinning machine. In this article, we chose a PCNFs membrane with an area of $352 \mathrm{~cm}^{2}$. In addition, the hierarchical PCNF films prepared by the method in this paper, it could adapt to mechanical deformations with bending and geometric cutting. 
(a)

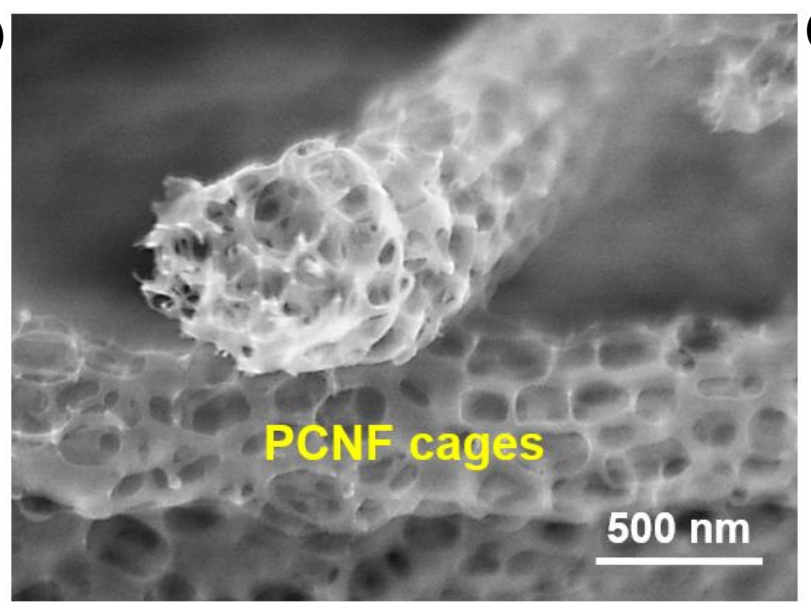

(b)

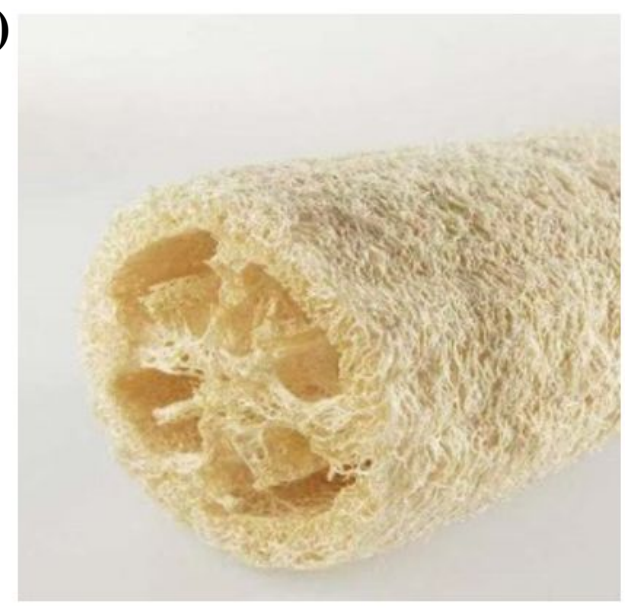

Figure S4. The cross-sectional morphology of (a) single PCNFs and (b) loofah.

The single PCNF had an intact fiber structure, in which hierarchical pore structure were connected to each other that offered a high degree of interoperability. Such PCNF with ordered hierarchical pores looked like loofah. 
(a)
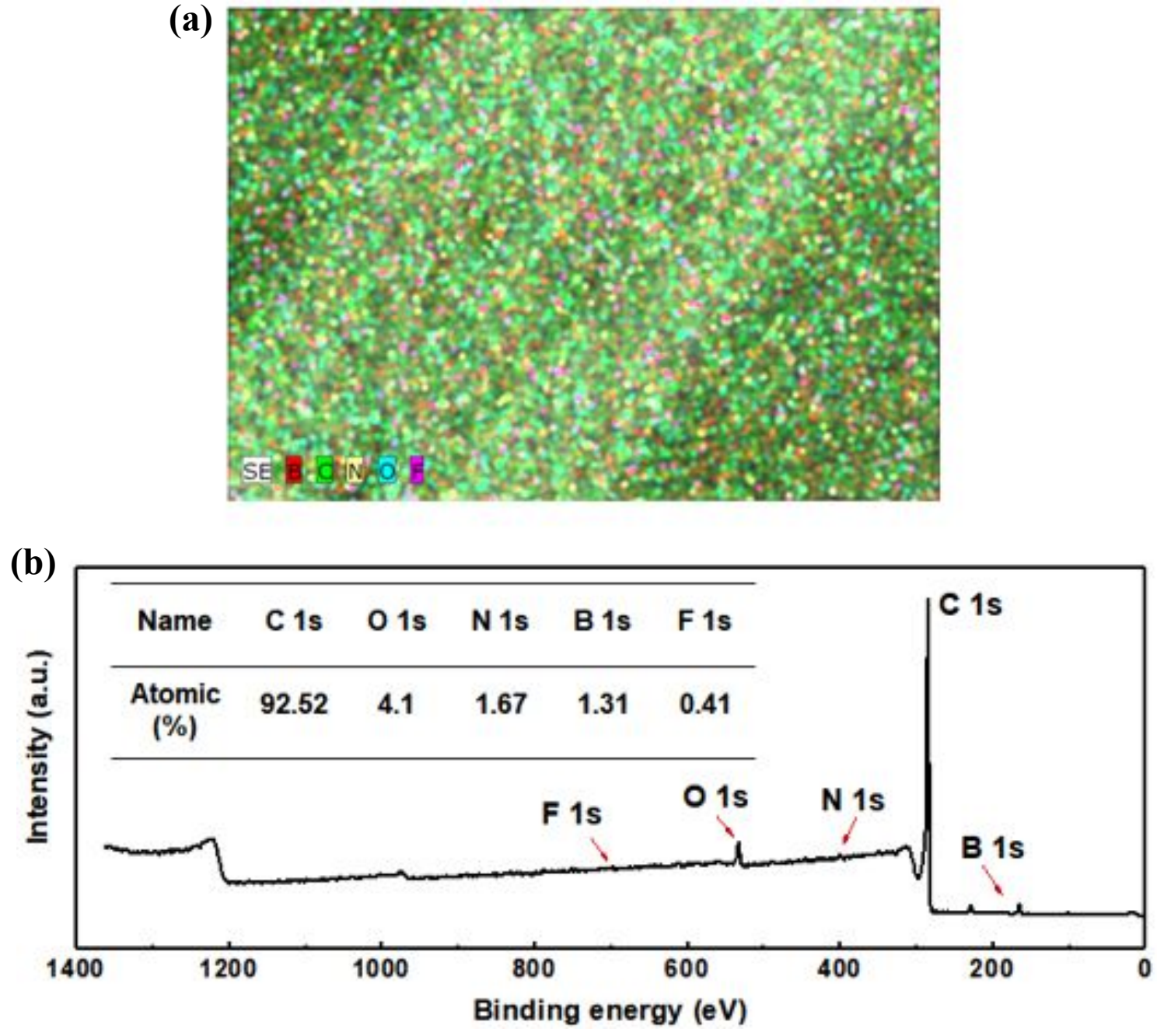

Figure S5. (a) EDS mapping and (b) XPS spectra of PCNFs.

(a) From the enlarged and detailed elemental distribution in PCNFs, the B, C, N, O and F were evenly dispersed throughout the nanofiber without agglomeration. (b) XPS analysis of PCNFs, the atomic percent of C, B, N, O, and F were $92.52 \%, 1.31 \%, 1.67 \%$, $4.1 \%$, and $0.41 \%$, respectively 

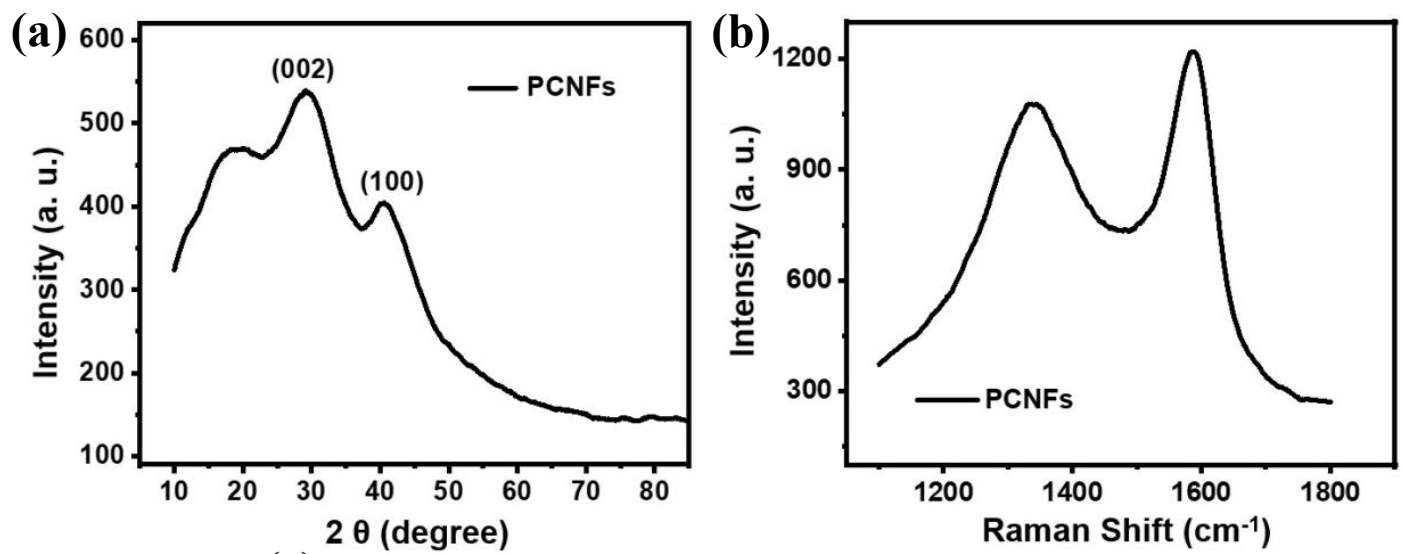

(c)

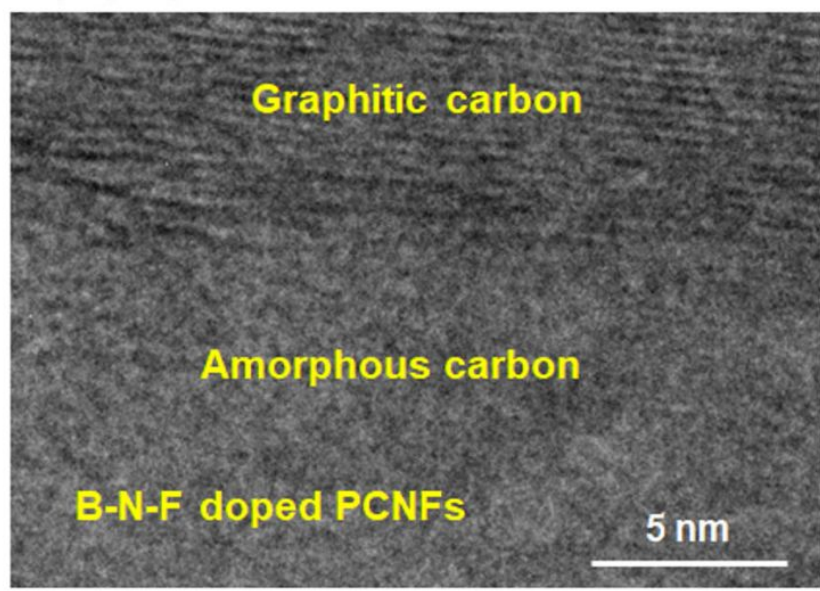

Figure S6. (a) XRD, (b) Raman spectra and (c) TEM images of PCNFs.

(a) From the XRD curves, the PCNFs showed a sharper and larger peak between 20$30^{\circ}$, indicating more graphite domains in the PCNFs. (b) It can be seen from the Raman spectrum that PCNFs had the amorphous carbon domains (D-bands) and the graphite crystalline structures (G-bands), and had a high degree of graphitization. (c) The PCNFs contained amorphous carbon domains and graphitic carbon domains. 
(a)

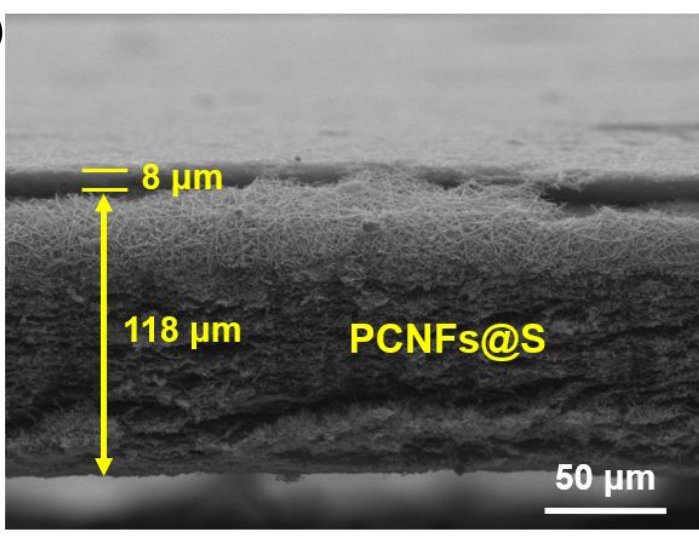

(c)

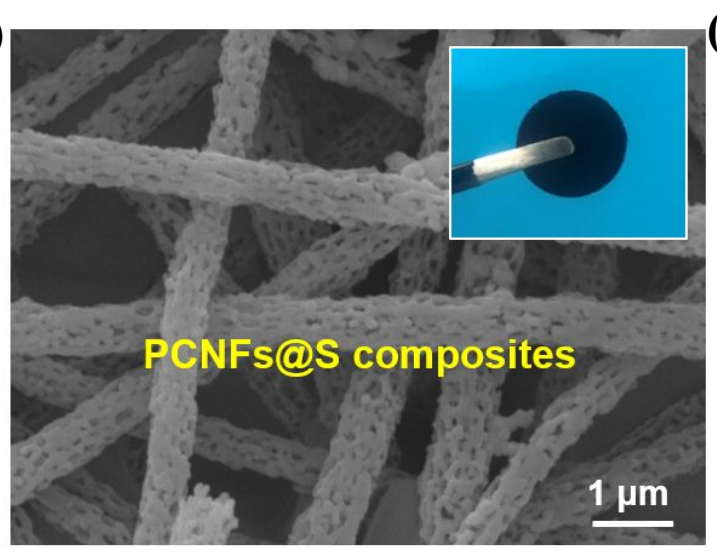

(b)

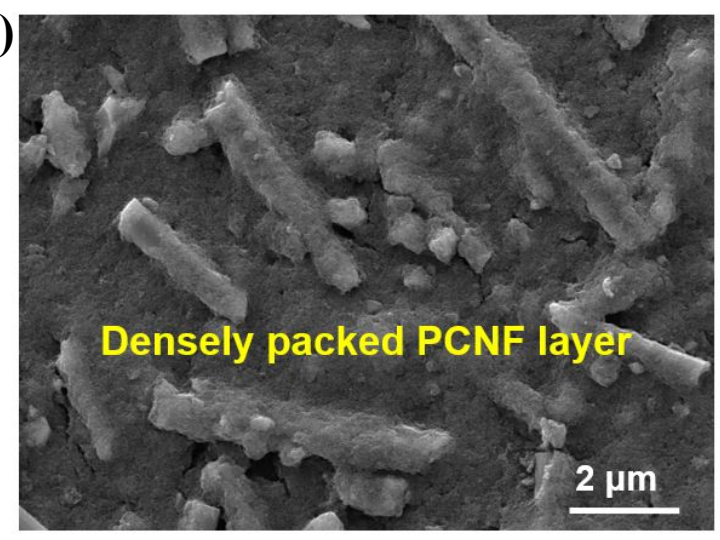

(d)

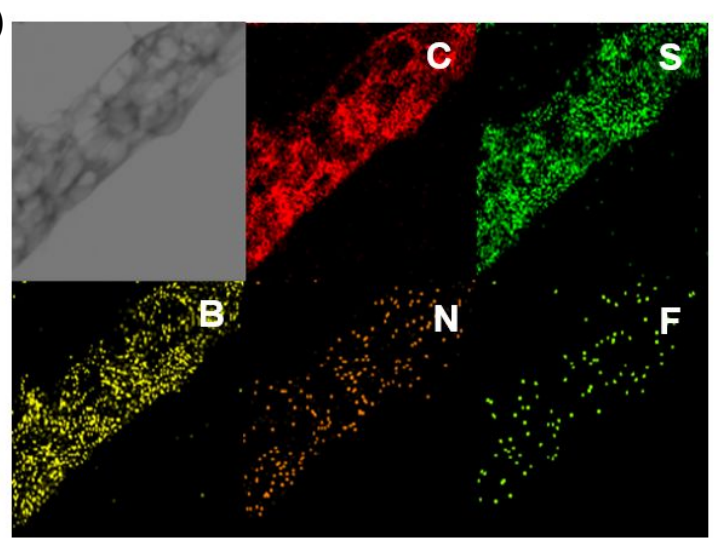

Figure S7. (a) A cross-sectional SEM image of the PCNFs@S electrode. Surface morphology of (b) the densely packed PCNF layer and (c) the PCNFs@S. (d) EDS mapping spectrum of PCNFs@S.

(a) According to actual needs, the thickness of the PCNFs@S film was $126 \mu \mathrm{m}$ in this paper, which included a thin $(8 \mu \mathrm{m})$ PCNF layer. (b) The thin PCNF layer that composed of $95 \mathrm{wt} . \%$ of the PCNF powder and $5 \mathrm{wt} . \%$ of block copolymer binder of PVDF, which was densely coated on the surface of the electrode. (c) Sulfur preferred to infiltrate into the macropores without forming many sulfur agglomerates, and the whole could be self-supporting. (d) The EDS spectra confirmed that sulfur was uniformly distributed into/onto PCNFs, while the doped elements of B, N, and F were well maintained. 

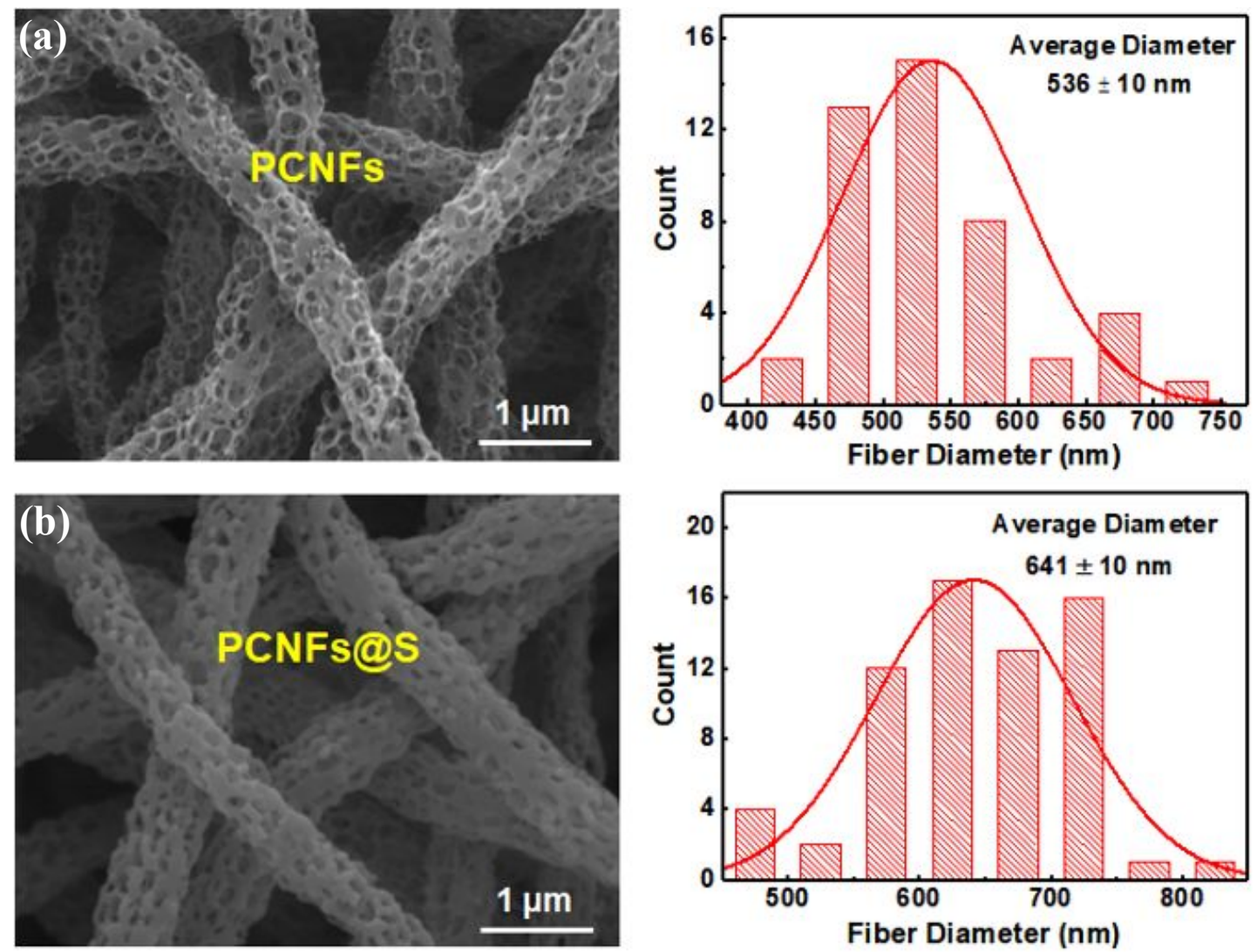

Figure S8. SEM figures and diameter histograms of (a) the PCNFs and (b)

\section{PCNFs@S.}

Compared the nanofiber before and after sulfur loading, the average fiber diameters of the (a) PCNFs and (b) PCNFs@S were 536 nm and 641 nm, and they had no obvious changes, which confirmed that sulfur preferred to be located into the pores without forming agglomerates in the surface of PCNFs. 
(a)

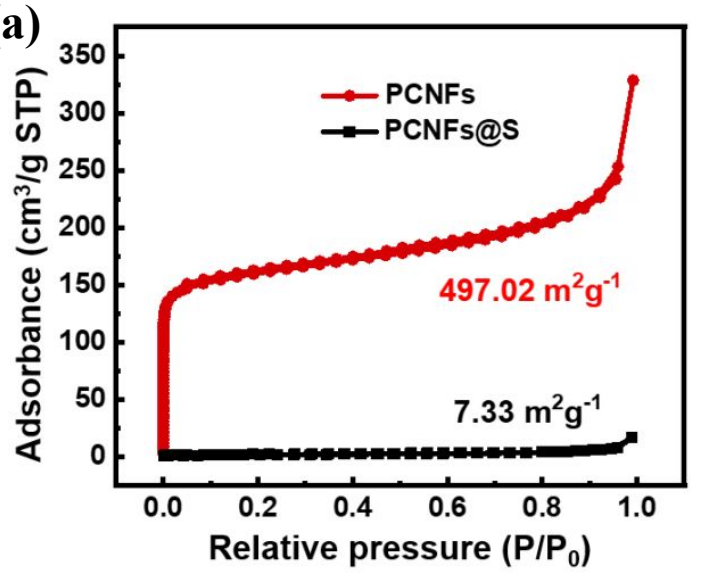

(c)

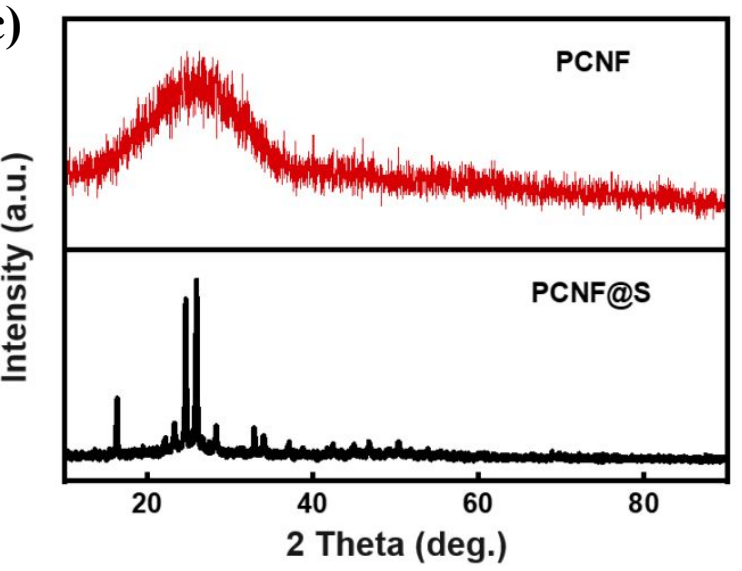

(b)

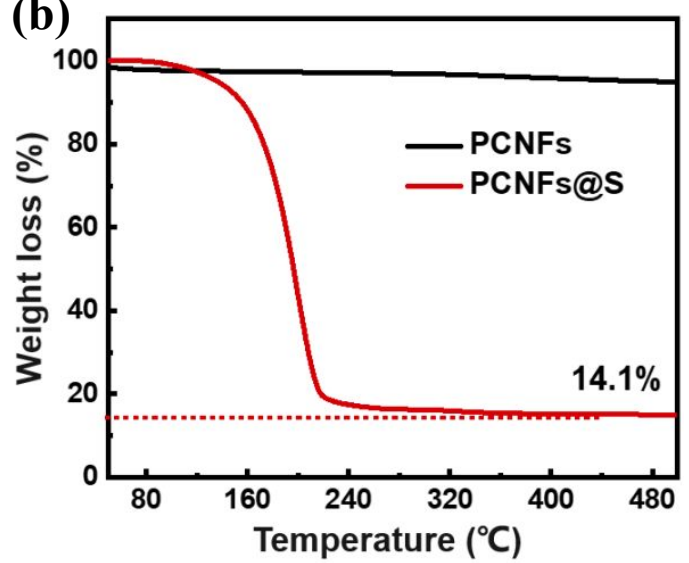

$4.1 \%$

$$
480
$$

$$
\text { . }
$$


(a)

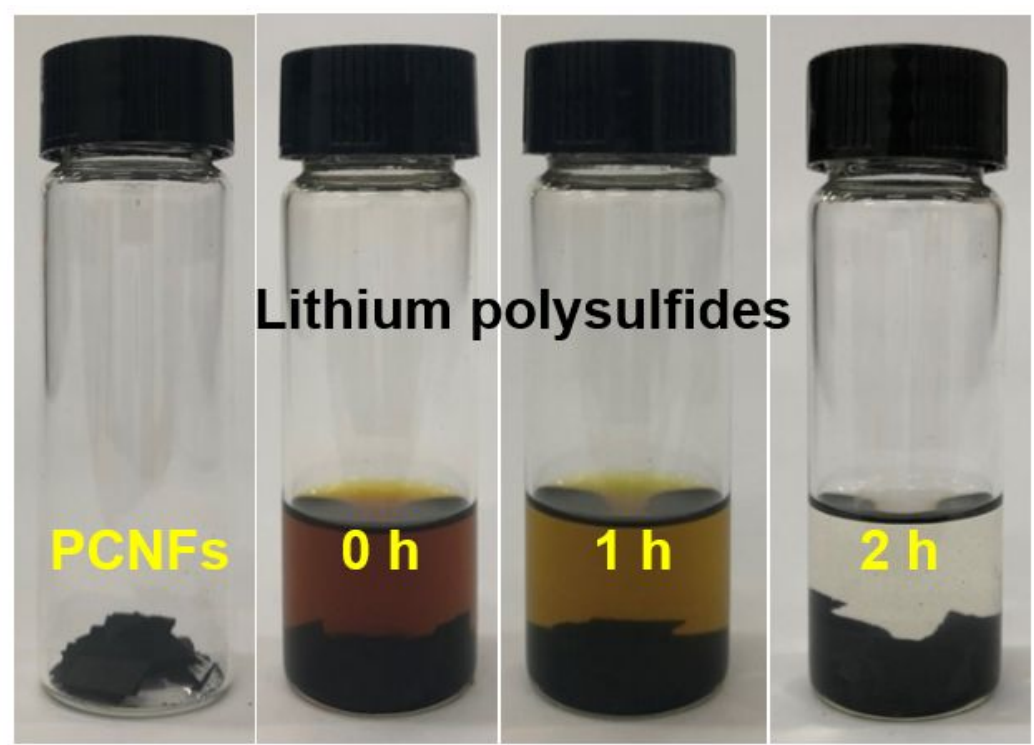

(b)

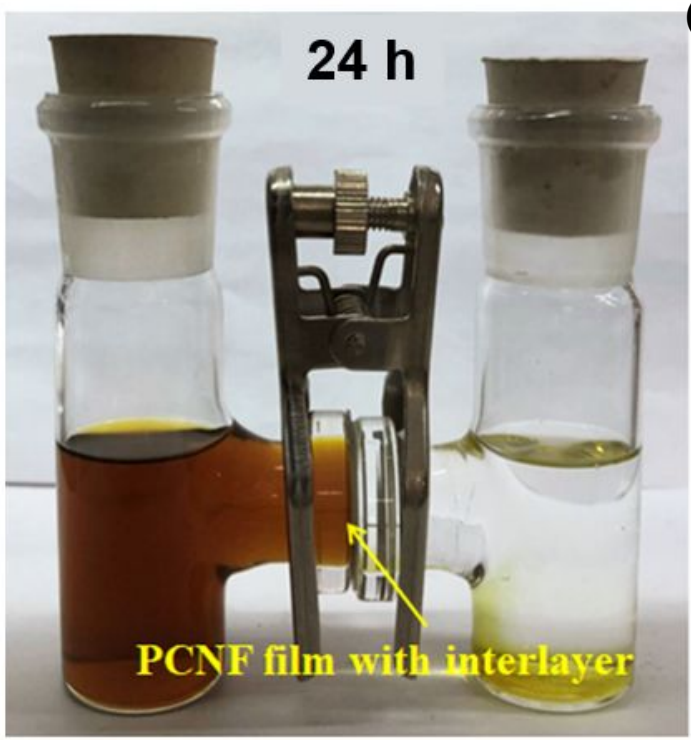

(c)

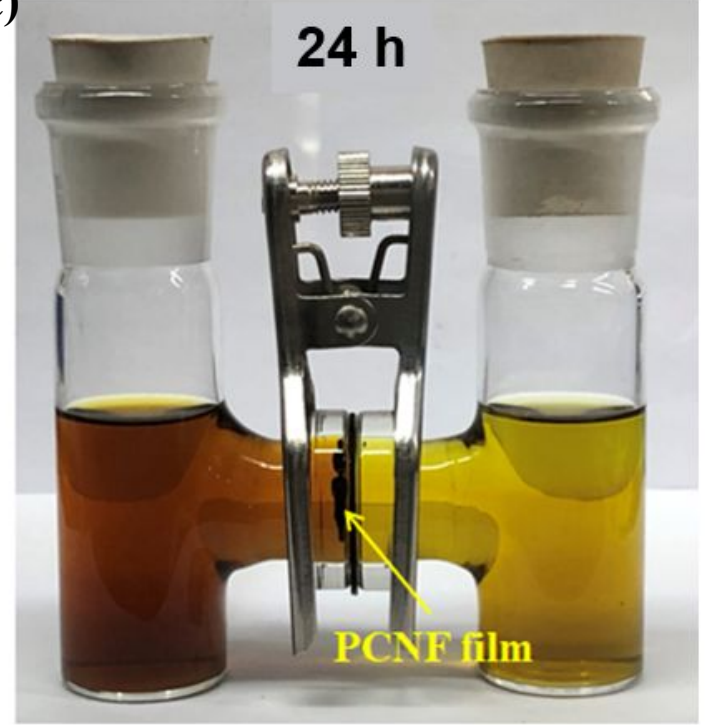

Figure S10. Optical photograph of soluble polysulfide (a) being adsorbed and (b-

c) infiltrated for $24 \mathrm{~h}$.

(a) Adding $20 \mathrm{mg}$ of PCNFs to $10 \mathrm{~mL}$ of $0.1 \mathrm{~mol} \mathrm{L-1} \mathrm{Li} \mathrm{Li}_{6}$ solution, the rapid color changes of the $\mathrm{Li}_{2} \mathrm{~S}_{6}$ solution from dark brown to transparent within $2 \mathrm{~h}$, indicating the such PCNF cages could efficiently adsorb soluble lithium polysulfides. (b) When used the PCNF film with the PCNF interlayer as the separator the right chamber became light yellow after 24 h. (c) In contrast, after removing the densely packed PCNF layer, the right chamber quickly became yellow, and the color deepened after $24 \mathrm{~h}$. 
(a)

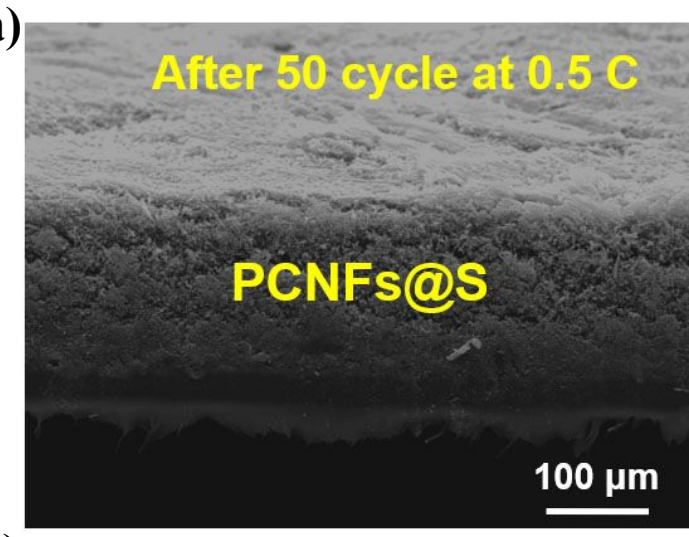

(c)

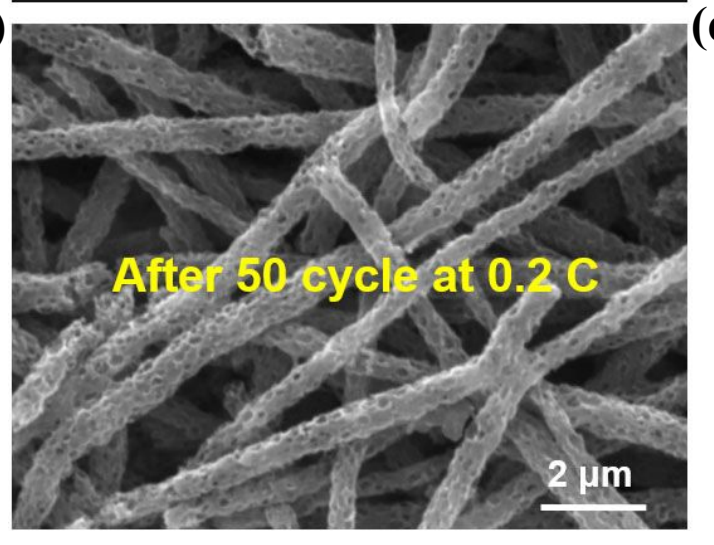

(b)

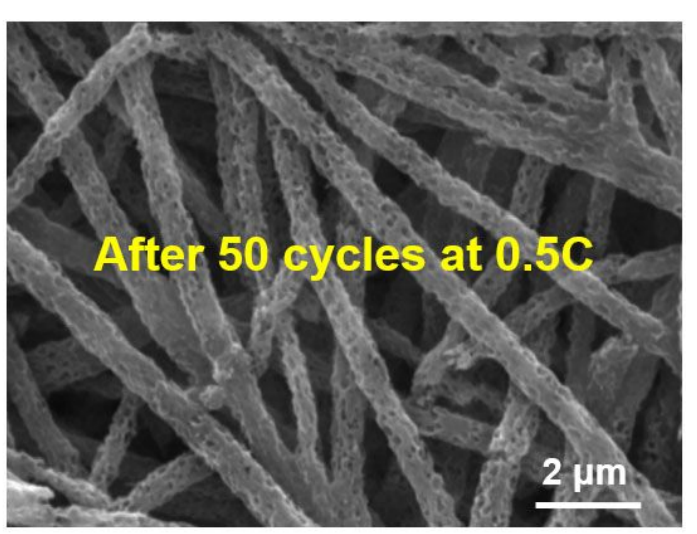

(d)
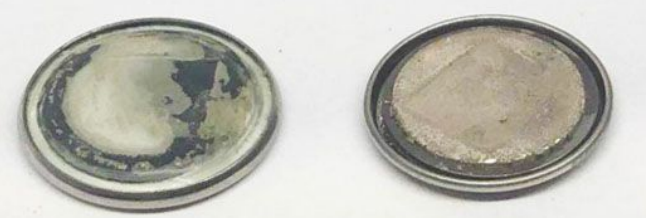

Li-metal anode

Figure. S11. Characterizations of (a-c) sulfur cathode and (d) Li-anode after 50 deep cycles at $0.5 \mathrm{C}$ and $0.2 \mathrm{C}$.

Whether it is from the cross-section (a) or the surface SEM image (b-c), one can know that the PCNFs@S cathode maintained its structure well without forming large sulfur clusters or breaking after 50 deep cycles at $0.5 \mathrm{C}$ and $0.2 \mathrm{C}$. (d) The Li-anode surface kept gray without being covered by yellow sulfur. 

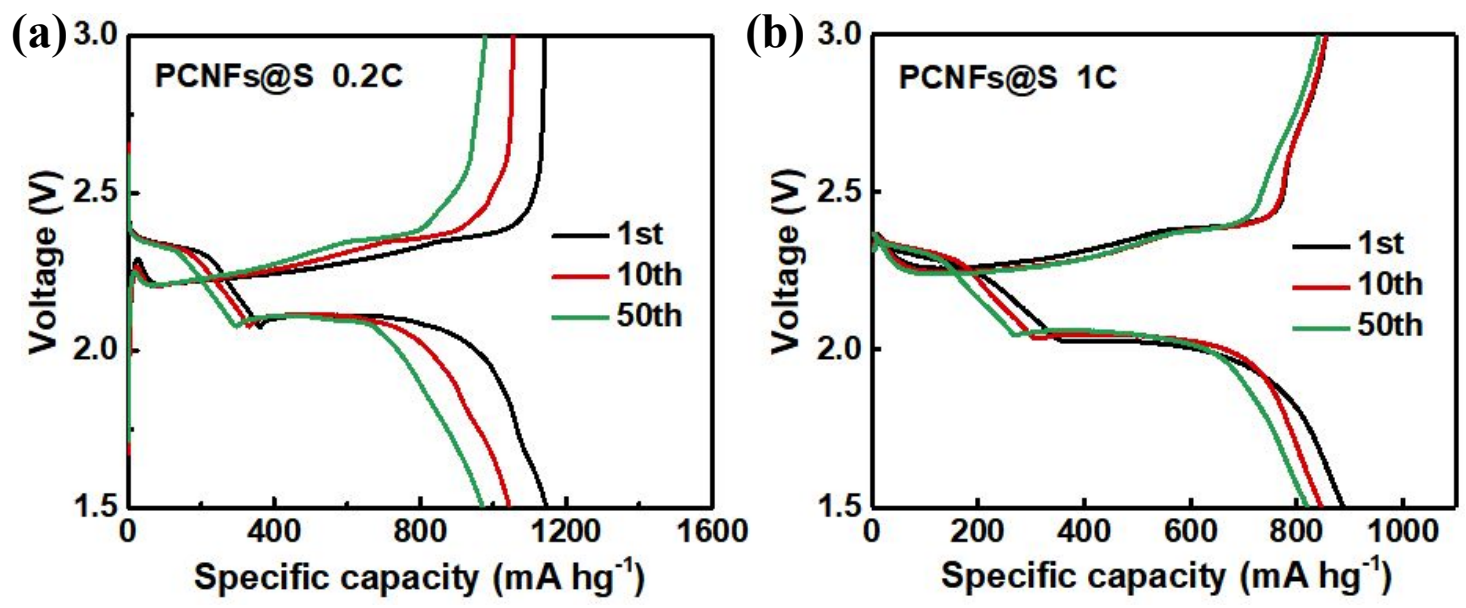

Figure. S12. (a) Galvanostatic discharge and (b) charge profiles of Li/PCNFs@S.

(a) At $0.2 \mathrm{C}$, the batteries showed a high initial capacity of $1162 \mathrm{~mA} \mathrm{~h} \cdot \mathrm{g}^{-1}$, and the retained capacity was as high as $939.6 \mathrm{~mA} \mathrm{~h} \cdot \mathrm{g}^{-1}$ over 50 cycles. (b) At $1 \mathrm{C}$, the batteries showed a high initial capacity of $899.2 \mathrm{~mA} \mathrm{~h} \cdot \mathrm{g}^{-1}$, and the retained capacity was $811 \mathrm{~mA}$ $\mathrm{h} \cdot \mathrm{g}^{-1}$ over 50 cycles. 